\title{
Os significados do pré-natal atribuído por gestantes realizado por enfermeiros
}

\author{
The meanings of prenatal assigned by pregnant women performed by nurses \\ Los significados de prenatal asignado por mujeres embarazadas realizado por enfermeras
}

Recebido: 10/05/2021 | Revisado: 16/05/2021 | Aceito: 18/05/2021 | Publicado: 06/06/2021

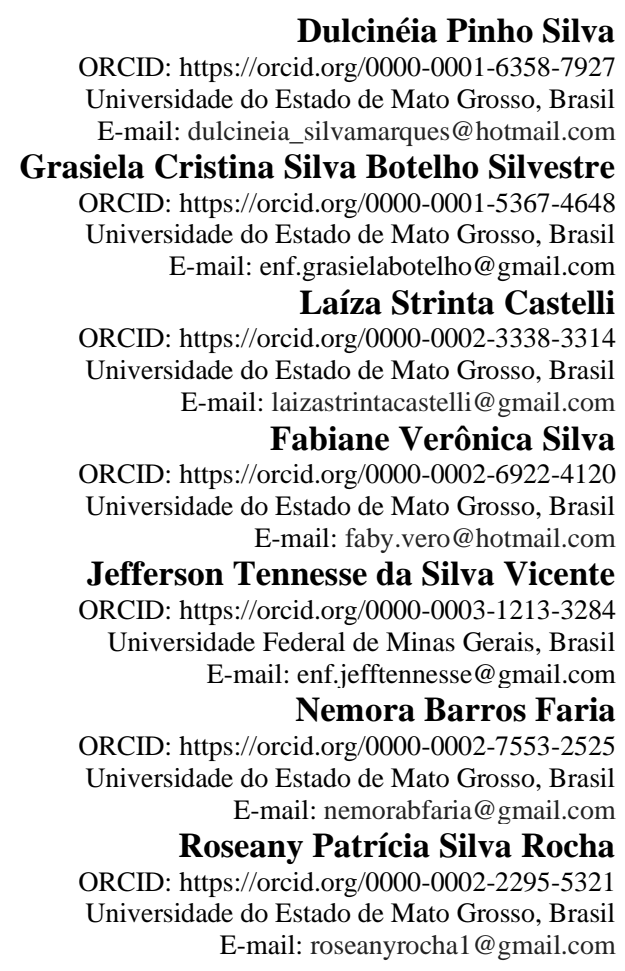

\section{Resumo}

Introdução: O acompanhamento de pré- natal deve pautar-se na qualidade da assistência, baseando-se na integralidade e humanização para promoção da saúde, prevenir de doenças ou agravos e recuperação da saúde materna e neonatal. A valorização da opinião da mulher acerca das decisões que envolvem seu plano terapêutico é relevante e oportuna, sendo assim, o presente artigo tem como objetivo compreender os significados atribuídos por gestantes ao pré-natal realizado por enfermeiros. Metodologia: Trata-se de estudo exploratório, descritivo, de abordagem qualitativa, com doze gestantes cadastradas em uma Estratégia Saúde da Família da região médio norte do estado de Mato Grosso. A coleta de dados deu-se por intermédio de entrevistas semiestruturadas, analisadas conforme a análise de conteúdo do tipo temática. Resultados: dos achados emergiram duas categorias temáticas: a) significando o período gestacional e o acompanhamento de pré-natal; e b) a qualidade da consulta de enfermagem no pré-natal. Conclusão: os resultados sugerem que o pré- natal realizado pelo profissional enfermeiro e a importância da qualidade do pré-natal foram considerados pelas mulheres como um processo importante e necessário. As mulheres sentiram-se motivadas pelo cuidado e atenção dos enfermeiros nas consultas, sanando suas inquietações. Além disso, robustece a discussão sobre iniciativas de empreendedorismo na enfermagem e o fortalecimento da categoria, e da identidade profissional, que vem assumindo espaços nos diversos serviços de saúde.

Palavras-chave: Gestantes; Cuidado pré-natal; Enfermeiros.

\begin{abstract}
Introduction: Prenatal care should be based on the quality of care, comprehensiveness and humanization for health promotion, prevention of diseases or injuries and recovery of maternal and neonatal health. The valorization of the woman's opinion about the decisions that involve her therapeutic plan is relevant and timely; therefore, this article aims to understand the meanings attributed by pregnant women to the prenatal care performed by nurses. Methodology: This is an exploratory, descriptive study, with a qualitative approach, with twelve pregnant women registered in a Family Health Strategy program in the northern region of the state of Mato Grosso, Brazil. Data collection took place through semi-structured interviews, analyzed according to thematic content analysis. Results: two thematic categories emerged from the findings: a) giving meaning to the gestational period and prenatal care; and
\end{abstract}


b) the quality of the prenatal nursing consultation. Conclusion: the results suggest that the prenatal care performed by a nurse and the importance of the prenatal care quality were considered as an important and necessary process. The women felt motivated by the nurses' care and attention in the consultations, especially when the professionals clarified the women's doubts. In addition, it fosters the discussion on nursing entrepreneurship initiatives and the strengthening of the professional category and professional identity, which have been constant in the various health services.

Keywords: Pregnant women; Prenatal care; Nurses.

\section{Resumen}

Introducción: La atención prenatal debe basarse en la calidad de la atención, basada en la integralidad y humanización para la promoción de la salud, la prevención de enfermedades o lesiones y la recuperación de la salud materna y neonatal. La valorización de la opinión de la mujer sobre las decisiones que involucran su plan terapéutico es relevante y oportuna, por lo que este artículo tiene como objetivo comprender los significados que le atribuyen las gestantes a los cuidados prenatales que realizan las enfermeras. Metodología: Se trata de un estudio exploratorio, descriptivo, con abordaje cualitativo, con doce gestantes inscritas en una Estrategia de Salud de la Familia en la región centro norte del estado de Mato Grosso. La recolección de datos se realizó a través de entrevistas semiestructuradas, analizadas según análisis de contenido temático. Resultados: de los hallazgos surgieron dos categorías temáticas: a) significado del período gestacional y atención prenatal; yb) la calidad de la consulta de enfermería prenatal. Conclusión: los resultados aseguran que la atención prenatal realizada por el profesional de enfermería es la importancia de la calidad de la atención prenatal y fue considerada por las mujeres como un proceso importante y necesario y se sintieron motivadas por el cuidado y atención de las enfermeras en las consultas, donde fue posible sanar las preocupaciones. Además, fortalece la discusión sobre iniciativas de emprendimiento en enfermería y el fortalecimiento de la categoría, e identidad profesional, que ha ido tomando espacios en los distintos servicios de salud.

Palabras clave: Mujeres embarazadas; Cuidado prenatal; Enfermeras.

\section{Introdução}

A gravidez é uma experiência única na vida da mulher. O ciclo gravídico denota alterações fisiológicas que envolvem todos os sistemas orgânicos, repercutindo mudanças físicas, sociais, emocionas somadas a ansiedade, medos e angustias. Portanto o conhecimento dessas transformações faz-se necessário, com a elucidação dos aspectos relevantes que permeiam o processo gravídico, instruções e ensinamentos que tangem a gestação, autocuidado, preparo para o parto e para a maternidade (Brasil, 2010).

A atenção pré-natal deve ser prestada com qualidade, integralidade e humanização para promover, prevenir e recuperar a saúde materna e neonatal. Os aspectos biológico, socioeconômico e cultural das mulheres e de seus neonatos devem ser considerados, com respeito aos direitos sexuais e reprodutivos e a subjetividade dos usuários na assistência à saúde (Brasil, 2006).

É na Atenção Básica através da Estratégia Saúde da Família (ESF) que as ações de pré-natal são implementadas, com a participação de uma equipe multidisciplinar. Tais ações devem ser acolhedoras, pautadas em ações educativas e preventivas, na detecção precoce de patologias e de condições de risco gestacional, formação de vínculo com o local do parto e o acesso ao serviço de saúde de qualidade, desde a Atenção Básica até os níveis de maior complexidade (Brasil, 2012a).

No âmbito da Atenção Básica o enfermeiro é um dos profissionais essenciais na assistência ao pré-natal. Sua formação é pautada em estratégias de promoção à saúde, prevenção de doenças e humanização do cuidado (Suhre, Pissaia \& Oreschi, 2017). Elabora o plano de assistência de enfermagem, de acordo com as necessidades identificadas e priorizadas, realiza as intervenções e orientações necessárias ao cuidado, referência a mulher a outros serviços e promove a interdisciplinaridade das ações com os demais membros da equipe de saúde (Brasil, 2012a).

Conforme legislação que rege o exercício da Enfermagem no Brasil, Decreto no 94.406/87 e Lei n ${ }^{\circ}$ 7.498, de 25 de junho de 1986, é privativo do enfermeiro, entre outros, realizar a consulta de enfermagem e a prescrição da assistência de enfermagem, ainda como como integrante da equipe de saúde, prescreve medicamentos previamente estabelecidos em 
programas de saúde pública e em rotina aprovada pela instituição de saúde, bem como realiza a assistência de enfermagem à gestante, parturiente, puérpera e ao recém-nascido.

A consulta pré-natal é um momento adequado para desenvolver ações educativas utilizando como ferramentas o diálogo, o vínculo e a valorização da opinião da mulher e seus acompanhantes. É importante que os profissionais de assistem a gestante estejam abertos ao fortalecimento de vínculo e ao esclarecimento de dúvidas sem julgamentos de valor. A avaliação constante da efetividade das estratégias e orientações fornecidas durante a consulta deve ser realizada, visto que a qualidade do serviço prestado é fator importante nos serviços de saúde (Pohlmann et al., 2016).

A valorização da opinião da mulher acerca das decisões que envolvem seu plano terapêutico é relevante e oportuna, desenvolvendo um cuidado integral e especifico as suas subjetividades. É importante que todos os profissionais de saúde, com destaque para o enfermeiro, estejam mais ávidos e competentes nas ações preventivas, educativas e de humanização no atendimento ao pré-natal. O vínculo de confiança entre a gestante e o enfermeiro é indispensável (Martins et al., 2015).

Através da concepção das gestantes na assistência recebida durante o pré-natal, elucidam-se aspectos positivos e negativos. As mulheres percebem a construção de vínculo entre as equipes de saúde e práticas de comunicação efetivas, ações que contribuem para a humanização da assistência. Por outro lado, o atendimento centrado no modelo biologicista e falhas na distribuição e implementação dos serviços ainda impera no modelo de Atenção Básica do Brasil (Santos et al., 2018). Percebese ainda a demora no atendimento, falta de planejamento na execução de atividades educativas, divulgação das ações ofertadas pela equipe de saúde, variação de dias e horários que favoreçam o atendimento a gestantes que trabalham (Oliveira, Brito \& Giotto, 2018).

Nota-se que existem limitações de estudos publicados no Brasil que remontam os significados e percepções da mulher à assistência ao pré-natal. Diante desse cenário, o objetivo deste estudo é compreender os significados atribuídos por gestantes ao pré-natal realizado por enfermeiros.

\section{Metodologia}

Trata-se de um estudo exploratório-descritivo de abordagem qualitativa (Estrela, 2018), realizado em uma ESF de um município da região médio norte do estado de Mato Grosso, Brasil. Utilizou-se como critério de elegibilidade: gestantes pertencentes a área de abrangência da ESF e que participam assiduamente do pré-natal e como critérios de exclusão gestante menor de idade, gestantes de alto risco e/ou que apresentasse algum distúrbio ou patologia que as impedisse de responder as perguntas.

Na ESF, estavam cadastradas dezoito (18) gestantes, no mês de setembro de 2017, os quais somente doze (12) foram atenderam aos critérios de elegibilidade no estudo, pois uma (1) era menor de idade, quatro (4) eram de alto risco e uma (1) quando foi aplicado o questionário já havia ocorrido o parto.

A coleta de dados foi realizada no segundo semestre de 2017, por meio de entrevista pré-agendadas e com auxílio de um questionário semiestruturado para nortear, com perguntas acerca das vivências, percepções, significados e sentimentos das gestantes frente ao pré-natal. Primeiramente foi explicado o objetivo da pesquisa e esclarecidas as dúvidas, $\operatorname{logo}$ após as gestantes assinaram o Termo de Consentimento Livre e Esclarecido (TCLE) confirmando a participação no estudo. Haja vista que foi abordado individualmente cada gestante para diminuir qualquer constrangimento. Todas as participantes receberam codinomes "G" seguido da numeração romana de acordo com a ordem das entrevistas, dessa maneira, garantindo sigilo e anonimato.

Os dados foram submetidos à análise de conteúdo do tipo temática, seguida pela organização dos dados, codificação, categorização e inferência (Bardin,2016). Destarte, emergiram duas categorias temáticas: Significando o período gestacional e 
o acompanhamento de pré-natal e A qualidade da consulta de enfermagem no pré-natal, as quais serão caracterizadas e discutidas com suporte na literatura científica.

Este estudo foi aprovado pelo Comitê de Ética em Pesquisa com seres humanos de uma universidade pública do estado de Mato Grosso em 31 de julho de 2017, conforme o que dispõe na resolução 466/2012, com o número do Certificado de Apresentação para Apreciação Ética (CAAE): 70367917.1.0000.5166.

\section{Resultados e Discussão}

A amostra foi composta por 12 gestantes, sendo 67,0\% (n=8) com idade entre 18 e 37 anos. Entre as gestantes, 42,0\% $(n=5)$ referiram possuir ensino médio completo, 50,0\% $(n=6)$ relataram encontrar-se em uma união estável e 67,0\% $(n=8)$ possuir vínculo empregatício. Quanto as variáveis gestacionais, 58,0\% $(n=7)$ eram multíparas e $42,0 \%$ ( $n=5)$ primigestas, 75,0\% (n=9) relataram não obter histórico de aborto, 50,0\% (n=6) referiram possuir de 1 e 5 filhos nascidos vivos (Tabela 1).

Tabela 1. Distribuição das gestantes $(n=12)$ segundo as variáveis sociodemográficas e gestacionais. Diamantino, Mato Grosso, 2017.

\begin{tabular}{|c|c|c|}
\hline Variáveis & $\mathbf{N}$ & $\%$ \\
\hline \multicolumn{3}{|c|}{ Sociodemográficas } \\
\hline \multicolumn{3}{|l|}{ Faixa etária } \\
\hline 18 a 30 anos & 8 & $67,0 \%$ \\
\hline 31 e mais & 4 & $33,0 \%$ \\
\hline \multicolumn{3}{|l|}{ Escolaridade } \\
\hline Analfabeto & 0 & 0,0 \\
\hline Ensino Fundamental Completo & 1 & $8,0 \%$ \\
\hline Ensino Fundamental Incompleto & 1 & $8,0 \%$ \\
\hline Ensino Médio Completo & 5 & $42,0 \%$ \\
\hline Ensino Médio Incompleto & 3 & $25,0 \%$ \\
\hline Ensino Superior Completo & 0 & 0,0 \\
\hline Ensino Superior Incompleto & 2 & $17,0 \%$ \\
\hline \multicolumn{3}{|l|}{ Estado civil } \\
\hline Casada & 2 & $17,0 \%$ \\
\hline Solteira & 4 & $33,0 \%$ \\
\hline Separada & 0 & 0,0 \\
\hline Divorciada & 0 & 0,0 \\
\hline Amasiada & 6 & $50,0 \%$ \\
\hline Viúva & 0 & 0,0 \\
\hline \multicolumn{3}{|l|}{ Ocupação } \\
\hline Estudante & 3 & $25,0 \%$ \\
\hline Desempregada & 1 & $8,0 \%$ \\
\hline Empregada & 8 & $67,0 \%$ \\
\hline \multicolumn{3}{|l|}{ Variáveis Gestacionais } \\
\hline \multicolumn{3}{|l|}{ Gestação } \\
\hline Primigesta & 5 & $42,0 \%$ \\
\hline
\end{tabular}


Multipara

Já teve aborto e ou natimorto

Sim

Não

Natimorto

Quantos Filhos Vivos

Nenhum

1 à 5

6 à 10
7

$58,0 \%$

$17,0 \%$

$75,0 \%$

$8,0 \%$

$1 \quad 8,0 \%$

TOTAL

5

$42,0 \%$

$6 \quad 50,0 \%$

$1 \quad 8,0 \%$

12

$100,00 \%$

Fonte: Autores.

A partir da análise dos dados emergiram duas categorias empíricas, a saber: Significando o período gestacional e o acompanhamento de pré-natal e A qualidade da consulta de enfermagem no pré-natal.

\subsection{Significando o período gestacional e o acompanhamento de pré-natal}

A gestação corresponde a um processo fisiológico, marcado por inúmeras mudanças físicas, psicológicas, sociais e emocionais, cujo significado é resultado das experiências vivenciadas por cada mulher, anteriores a esse período. Logo, a gestação tem potencial de transformação da mulher, podendo torná-la mais vulnerável ou mais fortalecida e amadurecida (Blank et al., 2019).

Os relatos das gestantes, evidenciam que o processo corresponde a um marco na vida da mesma;

"Gestação para mim é uma nova etapa de vida, é uma vida nova entre nós, gestação é algo muito bom de se sentir, poder saber que está gerando um ser vivo da gente. Acho que a melhor fase de vida da mulher, muito bom”. (G.VI)

"É gerar um filho, isso é uma benção de Deus, uma dádiva. Eu estou muito feliz por saber que tenho uma sementinha crescendo dentro de mim, uma felicidade, emoção inexplicável”. (G.IV)

"Para mim acho que gestação é um momento mágico que a mulher vive, uma nova fase que estou vivendo a minha vida, uma nova experiência”. (G. V)

A qual, mesmo não correspondendo a primeira gestação, as expectativas e experiências vivenciadas durante esse período tendem a (re) significar a maternidade e vida da mulher, portanto é indispensável que a gestante receba orientações eficientes para tornar o período gravídico mais tranquilo para si e para a família (Balssells et al., 2018), conforme apresentado no relato abaixo:

"É um momento muito lindo que passamos por transformações no corpo, à espera do bebê nos deixa ansiosa, eu estou vivendo com muita alegria, ele não foi planejado mais foi bem recebido por mim e meu esposo”. (G. VII)

Diante disso, o acompanhamento gestacional através das consultas de pré-natal constitui-se um conjunto de procedimentos clínicos e educativos capazes de orientar a evolução da gravidez, parto e cuidados ao recém-nascido para a 
gestante e família, co- responsabilizando-os por cada fase do processo (Santos et al., 2018; Dias, Anjos, Alves, Pereira \& Campos, 2018).

"Comecei no início da minha gestação. Tem sido algo muito importante para mim, tenho feito exames, ultrassons e
tomado medicamentos". (G.II) tomado medicamentos". (G.II)

"Desde o momento que fiquei sabendo que estava grávida, é muito bom, tô aprendendo como me cuidar e o cuidado com meu bebê, da saúde dele para nascer saudável”. (G.IV)

Os relatos corroboram com outros achados, cuja as percepções das gestantes retratam aspectos positivos em relação ao acompanhamento de pré-natal, como sendo um espaço de acolhimento e esclarecimento de dúvidas.

Portanto, ressalta-se a necessidade dos serviços de saúde, em especial os que se constituem como portas de entrada ao Sistema Único de Saúde se aperfeiçoarem como ambientes que possibilitem a mulher, tornar-se protagonista do momento que corresponde ao marco da sua vida, ofertando-lhe escuta ativa, informações, trocas de experiências, e o investimento em práticas coletivas, a fim de fortalecer as ações de atenção ao pré-natal, parto e nascimento. O pré-natal tem por objetivo a redução da morbimortalidade materna e infantil, com os desenvolvimentos de ações resolutivas e acolhedoras que visão a inserção das gestantes na rede de atenção à saúde (Guimarães, Parente, Guimarães \& Garnelo, 2018).

Para garantir a assistência integral as gestantes o Programa de Assistência à Saúde da Mulher (PAISM) foi criado em 1984, antes mesmo da criação do SUS, as políticas relacionadas à saúde da mulher evoluíram ao longo dos anos e com a consolidação do SUS (Nascimento et al., 2020).

A assistência pré-natal tem o intuito de identificar, de forma adequada e precoce, aquelas pacientes com mais probabilidade de apresentar evolução desfavorável e acolhê-las desde o princípio da gestação (Andrade, Santos, \& Duarte, 2019).

Sendo assim, à compreensão das gestantes acerca da importância do pré-natal na gestação, é relevante pois a maioria delas entendem que é um momento importante para o acompanhamento e desenvolvimento do bebê, bem como um momento oportuno e esclarecedor de suas dúvidas e anseios como pode se observar nas falas a seguir:

"Acompanhamento da unidade de saúde, dos profissionais, juntamente com a gestantes para saber como anda a saúde da mulher e do bebê" (G.III).

"É importante para sabermos se temos alguma doença e se tiver tratar durante o pré-natal, se o bebê tem alguma doença ou síndrome, e está preparada para receber o bebê com suas limitações”(G.VII).

"O pré-natal é muito importante ajuda muito a cuidar da sua saúde do bebê, e você pode tirar todas as suas dúvidas" (G.IX). "Pré-natal para mim, é um preparatório para o parto, é acompanhar cada semana do bebê e a saúde dele" (G.V).

Pode se observar, o quão importante é acompanhar a saúde dos recém-nascidos, além da importância do acompanhamento dos profissionais da saúde, pois com a realização do pré-natal a gestante passam a receber apoio para que esteja preparada a quaisquer intercorrências que poderão surgir durante a gestação, bem como estar preparada para o parto.

As consultas de pré-natal realizadas na Atenção Primária à Saúde apresentam-se como momento indispensável para ofertar orientações necessárias no período da gestação e puerpério, sendo ocasião indispensável para que a gestante possa 
revelar seu medos, anseios e dúvidas sobre esse momento singular dela mesma e do feto (Pereira, Ferreira, Araújo, Melo, \& Andrade, 2018).

Deste modo, o Ministério da Saúde orienta que para a realização de um pré-natal de qualidade é necessário um conjunto de recursos que compreende: recursos humanos, área física adequada, equipamentos e instrumentos mínimos, apoio laboratorial, material para registro, processamento, análise de dados e medicamentos. Porém, somente isso não garante que o pré-natal seja bem-sucedido, uma vez que para ter qualidade além destes aspectos é necessário um relacionamento de confiança entre os profissionais, a gestante e seus familiares (Brasil, 2014).

Diante disso, o enfermeiro na Unidade Básica de Saúde desempenha papel de destaque, visto que é o profissional com maior contato junto à comunidade, com a formação voltada ao cuidado o que direciona-o para uma atuação mais preventiva e habilitado para atender a mulher em qualquer fase da vida, e frente a gestação tende através do vínculo estabelecido antes do período favorecer a adesão aos cuidados exigidos pela gestação, sanando as dúvidas da gestante e inserindo- as como protagonista do processo (Martins et al., 2015).

Portanto, para as gestantes, o pré-natal é um momento esclarecedor e oportuno para aprendizado, haja vista que este orienta sobre as mudanças que ocorrem em decorrência da gestação possibilitando a inserção da família. Como nos traz nas falas a seguir:

\footnotetext{
"Entendo como um momento de descobertas de vários assuntos que ocorrem na gestação e a prevenção de risco a serem tomadas, ou melhor ser sujeitas no decorrer dos nove meses da gestação da mulher, com os profissionais capacitados para lhe atender e o apoio familiar" (G.XII).
}

"Algo de muita importância para mim e meu bebê. Porque além de saber como meu bebê está me ajuda entender um pouco mais" (G.II).

No entanto, algumas gestantes não compreendiam a importância da realização do pré-natal, evidenciando que a disparidade entre os atendimentos nos serviços públicos e privados corroboram para que as dúvidas dos acontecimentos no período não sejam devidamente esclarecidas, como disposto a seguir:

"Não entendo muito bem, fico meio na dúvida, porque eu vejo a diferença no privado e público. Tipo o privado ele examina todinho, já o público fica mais na pergunta, e escreve no cartão de gestante e só ouve o coração do bebê" (G.VIII). "Não entendo muita coisa. Porque muitas vezes temos respostas, outras não, por parte da médica” (G.XI).

É notório que a comunicação entre o profissional - paciente favorece o estabelecimento vínculo e consequentemente, maior adesão ao pré-natal, devido a confiança e segurança atribuída pela gestante ao profissional o que lhe possibilita maiores esclarecimento das dúvidas no decorrer das consultas (Afonso, Afonso, Jones, 2015).

Diante disso, fora observado que a maior parte das gestantes compreendem a importância da realização do pré-natal e um pequeno número de mulheres não compreendiam a importância do mesmo e fizeram algumas reclamações em relação a diferença do atendimento do sistema público e do privado e o esclarecimento de suas dúvidas, apesar de terem relatado gostar da enfermeira em si.

\subsection{A qualidade da consulta de enfermagem no pré-natal}

A assistência ao pré-natal é constituída por cuidados, condutas e procedimentos em razão da saúde da gestante e do feto, cuja a finalidade é detectar, curar ou controlar precocemente doenças, evitando complicações durante a gestação e parto. 
Garantindo então a saúde materna e fetal e consequentemente reduzindo os índices de morbimortalidade dos mesmos (Gomes et al., 2019).

O profissional enfermeiro nesse contexto é indispensável nessa assistência, visto que o mesmo estabelece com as gestantes uma relação de poder menos autoritário, pautando comumente seu plano assistencial na confiança e a escuta ativa sem pressa, possibilitando a liberdade de expressão, protagonismo da mulher no processo e inserção da família (Ramos et al., 2018).

A assistência humanizada é essencial em qualquer nível e formato de assistência, visto que a humanização dentro do contexto de saúde corresponde a um aspecto principal para a qualificação das ações em saúde, visto que a humanização possibilita o estabelecimento de vínculo entre os profissionais, serviços e pacientes (Dias et al., 2018).

Diante disso, os roteiros são essenciais para que todos os aspectos que englobe a saúde humana sejam atendidos. Assim, é indispensável que as consultas de pré-natal sejam humanizadas e qualificadas, abrangendo os aspectos sociais, epidemiológicos, antecedentes pessoais, ginecológico, sexuais e obstétricos e dados sobre a gestação atual. Para tal, o Ministério da Saúde garante ao profissional enfermeiro autonomia na solicitação de exames, encaminhamentos da gestante para outros profissionais da saúde, afim de garantir que o acompanhamento seja processado de forma integral (Dias et al., 2018, Melo et al., 2020).

É evidente que as gestantes compreendem a importância da consulta de pré-natal pelo enfermeiro, destacando que as orientações, dicas, esclarecimento de dúvidas, educações em saúde são fornecidas em sua maioria pelo mesmo, isso pois, o faz desse primeiro contato das gestantes com o profissional o estabelecimento de vinculo, transferindo tranquilidade no processo gestacional às gestantes (Dias et al., 2018, Melo et al., 2020). Portanto, é notório o sentimento de satisfação em relação ao atendimento ofertado pelo enfermeiro durante as consultas de pré-natal, evidenciado nas falas a seguir: "Acho que é fundamental na gestação, principalmente quando corre alguns riscos. Pois nesse caso temos uma ótima enfermeira
na unidade de saúde, além de ser uma profissional, é uma pessoa atenciosa, amiga e companheira. Sem contar que pergunta como
estamos, mede a barriga, escuta o coração, então não temos só uma profissional, temos aqui uma amiga" (G.VI).

"Passa segurança ajuda aos cuidados na gestação, tira minhas dúvidas, ela tem um bom diálogo" (G.I).

Vale ressaltar a importância e à legitimação das mulheres perante a consulta de enfermagem ao pré-natal, pois ao perceber o conhecimento técnico-científico e a condução do atendimento, pautado no respeito à vida, à dignidade e à promoção da integralidade do cuidar, as usuárias começaram a se sentir acolhidas e seguras no atendimento pelo enfermeiro, como remete as falas a seguir:

"[...] as pessoas da saúde têm sido muito atenciosas com as gestantes nesta unidade, principalmente a enfermeira, más os demais colaboram bastante [...]” (G.VI).

"Eu acho importante pois acho que o enfermeiro responde melhor as nossas dúvidas, do que o médico. E também por achar que os enfermeiros se esforçam mais nos estudos, eles parecem que tem um interesse maior na área”. (G. V)

A educação em saúde é entendida como pelas gestantes como fator essencial para que seja assegurado uma gestação, parto e puerpério sem complicações. Destarte é indispensável que o enfermeiro seja capacitado para prestar uma assistência qualificada e contínua, ampliando as ações educativas individuais e coletivas ao grupo de gestantes cadastradas e atendidas pelo serviço (Lemos \& Medeiros,2019; Sehnem et al., 2020). 
O conhecimento técnico- científico associado ao vínculo de confiança estabelecido pelas gestantes com o profissional enfermeiro durante o pré-natal, atribuído a este, um papel de profissional mais empático e resolutivo. Assim como, descrito nas falas a seguir:

"A enfermeira avalia o lado emocional da gestante, através de como passou a semana, se está tudo bem, se tem algo a reclamar sobre o atendimento" (G.VI).

"Sim, pois ela faz o acompanhamento perguntando, sobre o emocional e as vivencias se estão bem e o que fazer para melhorar" (G.III).

O relato demonstra o desenvolvimento de um atendimento humanizado e holístico, bem como a importância do conhecimento do contexto social e familiar vivenciado pela gestante, para a evolução de uma gravidez sem intercorrência, e/ ou compreensão da ocorrência de intercorrências no processo. Uma vez que é sabido que as mudanças emocionais do período podem refletir positiva ou negativamente no período.

Para além das condutas durante o atendimento, os aspectos inerentes ao serviço também corroboram para uma percepção significativa a respeito do pré-natal, como exposto nas falas:

"É bom pois tem o agendamento e é atendido cada um em seu horário" (G.III).

"O pré-natal, não tem tido demora no atendimento" (G.VII).

"O atendimento é ótimo não tem muita espera. Atendimento super rápido não tenho do que reclamar" (G.I).

Dentre as percepções negativas as algumas gestantes destacaram a demora na sala de espera para serem atendidas, ressaltando possuírem prioridade no atendimento. Assim como as falas a seguir:

"Por se tratar de uma gestante acho que o atendimento demora muito, como dizem que as gestantes têm prioridade a espera deveria ser menor" (G.V).

"Muito enrolado para o início principalmente pela parte da médica. Más quando começa é ligeira no atendimento" (G.XII).

Claramente, a satisfação das gestantes frente as consultas realizadas pelos enfermeiros, ocorrem devido ao diferencial da escuta atenta, abertura para o estabelecimento de vinculo e confiança, por se tratar-se de um espaço para esclarecimentos de dúvidas, educações em saúde e ressignificação do momento através da quebra de pré-conceitos estabelecidos pelas gestantes devido influencias do meio social (Lemos \& Medeiros,2019, Sehnem et al., 2020).

Portanto, a garantia de uma assistência qualificada significa prevenir, diagnosticar e tratar eventos indesejáveis que possam ocorrer durante a gestação, o enfermeiro assume importante papel ao desenvolver ações de saúde no pré-natal, prevenindo, protegendo, recuperando e promovendo a saúde da gestante e do feto. As gestantes foram acolhidas pelos profissionais de saúde, de maneira que se sentiram seguras e tiveram suas dúvidas esclarecidas, trazendo contentamento para elas. Dessa maneira, os enfermeiros devem atuar de forma que os cuidados do pré-natal ultrapassem a dimensão biológica, acompanhando as gestantes em todas suas emoções e vivencia. 
Como limitações do estudo tem-se o fato de não permitirem a generalização e ser estudado uma única unidade de saúde. Assim, sugere-se novos estudos no sentido de buscar os significados atribuídos por mulheres do pré-natal realizados por enfermeiros, para fortificar a assistência do cuidado integralizado.

\section{Conclusão}

Conclui-se que o pré-natal é considerado um processo importante para as mulheres deste estudo e elas conseguem entender a necessidade de realizá-lo. Os resultados aqui analisados revelam a importância da qualidade do pré-natal pois sua adequada estruturação e oferta têm resultados positivos na redução da morbimortalidade materna e infantil.

Nesse cenário, as mulheres tiram proveito das orientações recebidas, seja pelo fato delas se sentirem motivadas para o aprendizado ou pelo cuidado/atenção dos enfermeiros, que sanam inquietações e incertezas sobre a assistência e cuidados quando verbalizados.

Desta forma, para que as gestantes tenham uma boa adesão e melhor condução da assistência de pré-natal é relevante que haja acesso à variedade de informações, bem como diálogo sobre a aplicabilidade desses conhecimentos ao longo do processo gestacional e puerperal. A comunicação entre enfermeiro e a gestante é um valioso instrumento terapêutico para o fortalecimento dos vínculos, o que contribuirá para uma assistência humanizada e de qualidade.

As inferências acerca deste estudo constituem notáveis subsídios para a atuação de enfermeiros, sobretudo para a assistência ao pré-natal, com a perspectiva do cuidado integral da mulher. Além disso, robustece a discussão sobre iniciativas de empreendedorismo na enfermagem e o fortalecimento da categoria, e da identidade profissional, que vem assumindo espaços nos diversos serviços de saúde. Sugere-se a elaboração de pesquisas que enfoquem na qualidade de indicadores na assistência ao pré-natal e que fomentem a criação de estratégias para a melhor vínculo da gestante com o profissional enfermeiro.

\section{Referências}

Andrade, U. V., Santos J. B., \& Duarte C. A. (2019). Percepção da Gestante sobre a Qualidade do Atendimento Pré-Natal em UBS, Campo Grande, MS. Revista Psicologia e Saúde, 11(1), 53-61.

Afonso, J. A., Afonso, K. K. A., \& Jones, K. M. (2015). Percepção das gestantes frente ao pré-natal prestado pelo enfermeiro. Revista Brasileira de Pesquisa em Ciência da Saúde. 2(1), 22-26.

Balsells, M. M. D., Oliveira, T. M. F., Bernardo, E. B. R., Aquino, P. S., Damasceno, A. Castro, R. C. M. B., Lessa, P. R. A., \& Pinheiro, A. K. B. (2018). Avaliação do processo na assistência pré-natal de gestantes com risco habitual. Acta Paulista de Enfermagem, 31(3), $247-254$.

Bardin, L. (2016). Análise de conteúdo. Edições 70.

Blank, E. B., Soares, M. C., Cecagno, S., Ribeiro, J. P., Oliveira, S. G., \& Ferreira, J. B. (2019). Educational practices to (re)signify both delivery and birth in the look of puerperal women. Rev. Salusvita (Online), 38(3): 581-595.

Brasil. (2010). Assistência pré-natal: manual técnico. Ministério da Saúde. https://bvsms.saude.gov.br/bvs/publicacoes/cd04_11.pdf

Brasil. (2006). Pré-natal e Puerpério: atenção qualificada e humanizada - manual técnico/Ministério da Saúde, Secretaria de Atenção à Saúde, Departamento de Ações Programáticas Estratégicas - Brasília: Ministério da Saúde. http://bvsms.saude.gov.br/bvs/publicacoes/manual_pre_natal_puerperio_3ed.pdf

Brasil. (2012). Atenção ao pré-natal de baixo risco: série A: normas e manuais técnicos Cadernos de Atenção Básica, n 32 . Brasília (DF): Secretaria de Atenção à Saúde, Departamento de Atenção Básica. Recuperado de http://bvsms.saude.gov.br/bvs/publicacoes/cadernos_atencao_basica_32_prenatal.pdf

Brasil. (2014). Ministério da Saúde. Humanização do parto e do nascimento / Ministério da Saúde. Universidade Estadual do Ceará. 465. Cadernos HumanizaSUS; v. 4. https://www.redehumanizasus.net/sites/default/files/caderno_humanizasus_v4_humanizacao_parto.pdf

Decreto no. 94.406, de 8 de junho de 1987. (1989). Regulamenta a Lei no 7.498, de 25 de junho de 1986, que dispõe sobre o exercício da enfermagem, e dá outras providências. Diário Oficial da República Federativa do Brasil, http://www.cofen.gov.br/decreto-n-9440687_4173.html

Dias, E. G., Anjos, G. B., Alves, L., Pereira, S. N., \& Campos, L. M. (2018). Ações do enfermeiro no pré-natal e a importância atribuída pelas gestantes. Revista SUSTINERE, 6(1), 52-62. 
Research, Society and Development, v. 10, n. 6, e44210615937, 2021

(CC BY 4.0) | ISSN 2525-3409 | DOI: http://dx.doi.org/10.33448/rsd-v10i6.15937

Estrela, C. (2018). Metodologia Científica: Ciência, Ensino, Pesquisa. Editora Artes Médicas.

Gomes, C. B. A., Dias, R. S., Silva, W. G. B., Pacheco, M. A. B., Sousa, F. G. M., \& Loyola, C. M. D. (2019). Consulta de enfermagem no pré-natal: narrativas de gestantes e enfermeiras. Texto Contexto Enferm, 28: e20170544.

Guimarães, W. S. G., Parente, R. C. P., Guimarães, T. L. F., \& Garnelo L. (2018). Acesso e qualidade da atenção pré-natal na Estratégia Saúde da Família: infraestrutura, cuidado e gestão. Cad. Saúde Pública, 34(5): 00110417.

Lemos, A. P. S., \& Madeira, L. M. (2019). Care carried out by the obstetric nurse: The prenatal perception of the puperpera. Revista de Enfermagem do Centro oeste Mineiro. 9. e3281.

Martins, Q. P. M., Ferreira, G. S. M., Aragão, A. E. A., Gomes, F. M. A., Araújo, L. M., \& Ferreira, F. I. S. (2015). Conhecimentos das gestantes no pré-natal: evidências para o cuidado de enfermagem. S A N A R E, Sobral, 14(2), 65-71.

Melo, D. E. B., Costa e Silva, S. P., Matos, K. K. C., \& Martins, V. H. S. (2020). Prenatal nursing consultation: social representations of pregnant women. J Nurs. UFSM. 10 (e18), 1-18.

Nascimento, V. F., Silva T. F., Terças-Trettel, A. C. P., Hattori, T. Y., Lemes, A. G., \& Rodrigues, R. D. S. (2020). Perfil de orientações recebidas no prénatal no interior de Mato Grosso, Brasil. Revista Enfermería Actual, 39. 2020.

Oliveira, B. C. D., Brito, S. S., \& Giotto, A. C. Percepção das gestantes sobre o pré-natal. (2018). Rev Inic Cient Ext.; 1(2): 96-104.

Pohlmann, F. C., Kerber, N. P. C., Pelzer, M. T., Dominguez, C. C., Minasi, J. M., \& Carvalho, V. F. (2016). Prenatal care model in the far south of Brazil. Texto Contexto Enferm. 25(1): e3680013.

Pereira, D. O., Ferreira, T. L. S., Araújo, D. V., Melo, K. D. F., \& Andrade, F. B. (2018). Avaliação das consultas de pré-natal: adesão do pré-natal e complicações na saúde materno-infantil. Revista Ciência Plural. 2018; 3 (3):2-15.

Ramos, A. S. M. B., Almeida, H. F. R., Souza, I. B. J., Araújo, M. C. M., Pereira, P. S. L., \& Fontenele, R. M. (2018). A assistência pré-natal prestada pelo enfermeiro sob a ótica das gestantes. R. Interdisciplinar, 11(2), 87-96.

Santos, L. A. V., Lara, M. O., Lima, R. C. R., Rocha, A. F., Rocha, E. M., Glória, J. C. R., \& Ribeiro, G. C. (2018). História gestacional e características da assistência pré-natal de puérperas adolescentes e adultas em uma maternidade do interior de Minas Gerais, Brasil. Ciência Saúde Coletiva, 23(2), 617-625.

Sehnem, G. D., Saldanha, L. S., Airboit, J., Ribeiro, A. C., \& Paula, F. M. (2019). Consulta de pré-natal na atenção primária à saúde: fragilidades e potencialidades da intervenção de enfermeiros brasileiros. Revista de Enfermagem Referência, 5(1), e19050.

Suhre, P. B., Costa, A. E. K., Pissaia, L. F., \& Oreschi, C. (2017). Systematization of nursing assistance: perceptions from pregnant women monitored at a basic health unit. Rev Ciênc Saúde, 5(1):20-31. 